Article

\title{
Towards Experiments to Test Violation of the Original Bell Inequality
}

\author{
Andrei Khrennikov ${ }^{1, *}$ and Irina Basieva ${ }^{2,3}$ \\ 1 International Center for Mathematical Modeling in Physics, Engineering, Economics, and Cognitive Science, \\ Linnaeus University, 35195 Växjö, Sweden \\ 2 Prokhorov General Physics Institute, Vavilov str. 38D, 119991 Moscow, Russia; Irina.Basieva@gmail.com \\ 3 Department of Psychology, University of London, London WC1E 7HU, UK \\ * Correspondence: Andrei.Khrennikov@lnu.se
}

Received: 16 February 2018; Accepted: 11 April 2018; Published: 13 April 2018

check for updates

\begin{abstract}
The aim of this paper is to attract the attention of experimenters to the original Bell (OB) inequality that was shadowed by the common consideration of the Clauser-Horne-Shimony-Holt (CHSH) inequality. There are two reasons to test the OB inequality and not the $\mathrm{CHSH}$ inequality. First of all, the OB inequality is a straightforward consequence to the Einstein-Podolsky-Rosen (EPR) argumentation. In addition, only this inequality is directly related to the EPR-Bohr debate. The second distinguishing feature of the $\mathrm{OB}$ inequality was emphasized by Itamar Pitowsky. He pointed out that the OB inequality provides a higher degree of violations of classicality than the CHSH inequality. For the $\mathrm{CHSH}$ inequality, the fraction of the quantum (Tsirelson) bound $Q_{\mathrm{CHSH}}=2 \sqrt{2}$ to the classical bound $C_{\mathrm{CHSH}}=2$, i.e., $F_{\mathrm{CHSH}}=\frac{Q_{\mathrm{CHSH}}}{C_{\mathrm{CHSH}}}=\sqrt{2}$ is less than the fraction of the quantum bound for the $\mathrm{OB}$ inequality $Q_{\mathrm{OB}}=\frac{3}{2}$ to the classical bound $C_{\mathrm{OB}}=1$, i.e., $F_{\mathrm{OB}}=\frac{Q_{\mathrm{OB}}}{C_{\mathrm{OB}}}=\frac{3}{2}$. Thus, by violating the $\mathrm{OB}$ inequality, it is possible to approach a higher degree of deviation from classicality. The main problem is that the OB inequality is derived under the assumption of perfect (anti-) correlations. However, the last few years have been characterized by the amazing development of quantum technologies. Nowadays, there exist sources producing, with very high probability, the pairs of photons in the singlet state. Moreover, the efficiency of photon detectors was improved tremendously. In any event, one can start by proceeding with the fair sampling assumption. Another possibility is to use the scheme of the Hensen et al. experiment for entangled electrons. Here, the detection efficiency is very high.
\end{abstract}

Keywords: original Bell inequality; preparation of singlet states; possible experimental test

\section{Introduction}

In his paper [1] (see also [2]), Bell proposed the probabilistic test based on the EPR-argument [3]. The problem under consideration can be formulated as follows. Einstein, Podolsky, and Rosen proved that quantum mechanics (QM) is incomplete, since its formalism does not represent the EPR elements of reality. Suppose one wants to construct a subquantum theory completing QM. Such a theory should match statistical predictions of QM and, at the same time, it should describe EPR's elements of reality. Can such a theory be local? (as EPR hoped).

Bell proposed a test based on an inequality for correlations. This inequality will be called the original Bell inequality (OB inequality). This inequality was proved under the following crucial assumption about coupling the Bell model with hidden variables and the EPR elements of reality.

For the singlet state (as for the original EPR state), spin projections are EPR's elements of reality. These elements of reality are equal to measurement outcomes (elements of reality for $S_{2}$ are measurement outcomes for $S_{1}$ ). Hence, values of variables of a subquantum theory beyond the singlet state can be identified 
with possible outcomes of measurements. Therefore, for the singlet state, subquantum and quantum correlations can be identified (see Appendixes B and C for further discussion).

However, this beautiful theoretical scheme supporting nonlocal hidden variable theories did not match the experimental framework of that time, since the degree of (anti-)correlations (for the same setting on both sides) was not so high. This problem was solved by transition from the OB inequality to the $\mathrm{CHSH}$ inequality [4] or the similar inequalities: the $\mathrm{CH} 74$ inequality $[5,6]$ or the Eberhard inequality [7] (see [8] for comparison of these inequalities). Derivations of such inequalities are not based on the assumption of perfect (anti-) correlations. (For convenience, later, we shall compare the OB inequality only with the CHSH inequality, but a similar comparison can be done for other "CHSH-like inequalities" as the CH74 inequality and the Eberhard inequality.) The foundational difference between the $\mathrm{OB}$ and CHSH-like inequalities is discussed in Appendix B; see also Appendix C for the general discussion about Bell type inequalities and interpretations of quantum mechanics.

Although the authors think that only a violation of the OB-inequality can be used as the argument in favor of quantum nonlocality, this viewpoint does not match the conventional views. Therefore to stimulate experimenters to perform experiments to violate the OB inequality, we want to highlight that, as was stressed by Itamar Pitowsky [9], the OB inequality provides a higher degree of violations of classicality than the $\mathrm{CHSH}$ inequality. For the $\mathrm{CHSH}$ inequality, the fraction of the quantum (Tsirelson) bound

$$
Q_{\text {Tsirelson }}=2 \sqrt{2}
$$

to the classical bound $C_{\mathrm{CHSH}}=2$, i.e.,

$$
F_{\text {Tsirelson }}=\frac{Q_{\mathrm{CHSH}}}{C_{\mathrm{CHSH}}}=\sqrt{2}
$$

is less than the fraction of the quantum bound for the $\mathrm{OB}$ inequality

$$
Q_{\text {Pitowsky }}=\frac{3}{2}
$$

to the classical bound $C_{\mathrm{OB}}=1$, i.e.,

$$
F_{\text {Pitowsky }}=\frac{Q_{\mathrm{OB}}}{C_{\mathrm{OB}}}=\frac{3}{2}
$$

Thus, by violating the OB inequality, it is possible to approach a higher degree of deviation from classicality (see Appendix A for Pitowsky's comparison of measures of nonclassicality given by quantities $F_{\text {Tsirelson }}$ and $\left.F_{\text {Pitowsky }}\right)$. However, the main message of Pitowsky was not just that $F_{\text {Pitowsky }}>F_{\text {Tsirelson, }}$ but that, for multi-dimensional generalizations of the OB inequality,

$$
F_{\text {Pitowsky }} \rightarrow \infty \text {. }
$$

(However, for multi-dimensional generalizations of the $\mathrm{CHSH}$-like inequalities, $F_{\text {Tsirelson }}=K_{G}(n)$, where $K_{G}(n)$ is the Grothendieck constant of the order $n$, and, as was shown by Grothendieck, there exists

$$
\lim _{n \rightarrow \infty} K_{G}(n)=K_{G}
$$

the Grothendieck constant, see Appendix A).

Thus, by appealing to multi-dimensional analogs of the OB inequality, experimenters can, in principle, approach an arbitrary large value of the "quantum/classical fraction".

The main problem for performing an experimental test is that the $\mathrm{OB}$ inequality is derived under the assumption of perfect (anti-) correlations. Therefore, it was impossible to perform experiments to check violation of the OB inequality. However, the last few years were characterized by the amazing development of quantum technologies. Technological improvements led to the loophole free tests of 
the Bell-type inequalities (It may be interesting for the reader that the weblinks to the video-records of the talks of the leaders of all these experimental groups accompanied with the talks of Gregor Weihs and two talks of Philippe Grangier (at the special session BIG EVENT: Final Bells test, at the conference Quantum and Beyond, Växjö, Sweden, June 2016) can be found at the webpage of one of the authors of this paper: https://nu.se/en/staff/andrei.khrennikov/) (the CHSH, Eberhard, and Clauser-Horne inequalities) [10-12] (see also [13-18] for previous steps towards these long-aspired experiments) (As was expected by Bell, these experiments did not change the views of those who did not accept the conventional interpretation of experimental outputs, see, e.g., [19]).

One possibility to test violation of the $\mathrm{OB}$ inequality is to follow the quantum optics path initiated by Aspect [14]. Nowadays, there exist sources producing with very high probability the pairs of photons in the singlet state. Moreover, the efficiency of photon detectors was improved tremendously. Therefore, one can hope to violate the OB inequality, although this is still the real challenge (see Section 6). In any event, one can proceed under the fair sampling assumption, i.e., to solve first the problem of (anti-) correlations.

Another possibility is to test the $\mathrm{OB}$ inequality by using the scheme of the Hensen et al. experiment [10]. This experimental scheme does not suffer from inefficiency of detection. However, it seems that the quality of preparation of the singlet state is still insufficient to perform the experimental test to violate the $\mathrm{OB}$ inequality (see Section 6).

This paper is a short review based on the results of Pitowsky [9], Ryff [20], and Larsson [21]. Its aim is to collect these results in one text and consider experimental consequences of a combination of the results from Ryff [20] and Larsson [21] in the light of recent tremendous achievements of modern quantum information technologies.

In Section 3, we present probabilistic calculations to estimate the probability of preparation of the singlet state that is sufficient to test violation of the OB-inequality under the assumption of $100 \%$ of the detection efficiency. Theorem 2 implies that experimenters have to be able to prepare an ensemble in which more than $75 \%$ of pairs are in the singlet state (see also Ryff's paper [20]). Thus, the existing photon sources of high quality provide the possibility to test the OB inequality, at least under the assumption of fair sampling. In Section 4, we present probabilistic calculations to estimate the minimal efficiency of detection that is sufficient to test violation of the OB-inequality under the assumption of $100 \%$ fidelity in preparation of the singlet state. By Theorem 3, the efficiency of the joint detection should be higher than $88,9 \%$ (see Larsson's paper [21] for the original derivation of this bound). In addition, finally, in Section 5, we combined the results of Sections 3 and 4 . By combining $98 \%$ level of anti-correlations with $90 \%$ level of detection efficiency, one can test violation of the OB inequality.

We remark that generalized (perturbed) Bell's inequalities that are similar to inequalities obtained in Theorems 2-4 were actively used by one of the coauthors in foundational studies [22-25].

Successful experimental testing of violation of the OB inequality would be an important (although very challenging) contribution to clarification of quantum foundations.

\section{Classical and Quantum Bounds for the Original Bell Inequality}

We proceed in accordance with Bell's paper [1]. Let $p$ be a probability measure on the space of hidden variables $\Lambda$. (Bell used the symbol $\rho$.) We model measurements on a pair of systems $S_{1}$ and $S_{2}$ with the aid of random variables $A_{s}(\lambda)$ and $B_{s}(\lambda)$, where the parameter $s$ labels settings of measurement devices, $s=a, b, c$.

Consider correlations of these random variables given by the integrals:

$$
P(a, b)=\int_{\Lambda} A_{a}(\lambda) B_{b}(\lambda) d p(\lambda)
$$

It is assumed that these random variables take values \pm 1 and that the random variables corresponding to measurements on $S_{1}$ and $S_{2}$ are anti-correlated: 


$$
P(a, a)=\int_{\Lambda} A_{a}(\lambda) B_{a}(\lambda) d p(\lambda)=-1 .
$$

Under these assumptions, Bell derived [1,2] the following inequality:

$$
|P(a, b)-P(a, c)|-P(b, c) \leq 1
$$

(see also Section 3 for details). We call it the original Bell inequality or OB inequality.

This hidden variable model was confronted with spin measurements represented in QM by the spin operators $\sigma \cdot s$. In this case, $s$ is the unit vector in $\mathbf{R}^{3}$ representing the axis of spin projection. Thus, pairwise correlations for spin operators are compared with correlations for random variables. To distinguish measurements on systems $S_{1}$ and $S_{2}$, we shall use symbols $\sigma_{1} \cdot a$ and $\sigma_{2} \cdot b$.

The $\mathrm{OB}$ inequality implies that, for classical correlations, the upper bound $C_{\mathrm{OB}}$ for the expression $\Delta=|P(a, b)-P(a, c)|-P(b, c)$ equals one. Now, consider the the quantum case. To get perfect anti-correlations, we proceed with the singlet state

$$
\Psi=(|+-\rangle-|-+\rangle) / \sqrt{2} .
$$

For this state, we have

$$
P_{Q}(a, b)=\left\langle\sigma_{1} \cdot a \otimes \sigma_{2} \cdot b\right\rangle=-\langle a \mid b\rangle .
$$

One can find the quantum bound for the expression

$$
\Delta_{Q}(a, b, c)=\left|P_{Q}(a, b)-P_{Q}(a, c)\right|-P_{Q}(b, c)=|\langle a \mid b\rangle-\langle a \mid c\rangle|+\langle b \mid c\rangle .
$$

Theorem 1. $Q_{\mathrm{OB}}=\max _{a, b, c} \Delta_{Q}(a, b, c)=\frac{3}{2}$.

Proof. Under the suitable parametrization $\Delta_{Q}(a, b, c)$ can be represented as

$$
\Delta_{Q}\left(\phi_{1}, \phi_{2}, \theta\right)=2\left|\sin \phi_{1} \sin \phi_{2} \sin \theta\right|+1-2 \sin ^{2} \phi_{1} .
$$

It is easy to find that the maximal value of this function equals to $3 / 2$.

Consider, for example, three vectors in the same plane, $a=(1,0), b=(1 / 2,-\sqrt{3} / 2)$, $c=(-1 / 2,-\sqrt{3} / 2)$. Then, $P(a, b)=-\langle a \mid b\rangle=-1 / 2, P(a, c)=-\langle a \mid c\rangle=1 / 2, P(b, c)=-\langle b \mid c\rangle=-1 / 2$. Hence, $\Delta_{Q}(a, b, c)=3 / 2$.

Hence, we proved the equality (4), $F_{\mathrm{OB}}=3 / 2$.

Itamar Pitowsky [9] presented the same argument by using a slight modification of the OB inequality (7).

\section{Original Bell Inequality: Taking into Account Imperfection of Anti-Correlations}

Here, we proceed in Bell's framework based on classical probability under the assumption that the random variables corresponding to measurements on $S_{1}$ and $S_{2}$ are anti-correlated. As Bell pointed out, this is possible only if the following equality holds

$$
A_{a}(\lambda)=-B_{a}(\lambda),
$$

except a set of measure zero. Bell derived inequality (7) under this assumption of perfect (up to measure zero) anti-correlation. It is easy to modify this equality under assumption of imperfect anti-correlations. Here, we follow the original paper [20], but we proceed in measure theoretic framework. Using the frequentist approach (as in paper [20]) has been objected to by a few authors (see, e.g., [22]).

Suppose that, for each $a$, there exists a subset $\Lambda_{a}$ of $\Lambda$ such that Equation (11) holds for all $\lambda$ from $\Lambda_{a}$ and, for the set $\Lambda_{a}^{\prime}=\Lambda \backslash \Lambda_{a}$, we have: 


$$
p\left(\Lambda_{a}^{\prime}\right) \leq \epsilon
$$

Since random variables are dichotomous, on the set $\Lambda_{a}^{\prime}$

$$
A_{a}(\lambda)=B_{a}(\lambda) .
$$

Now, on $\Lambda_{b}$, we have:

$$
\begin{gathered}
A_{a}(\lambda) B_{c}(\lambda)-A_{a}(\lambda) B_{b}(\lambda)=A_{a}(\lambda) A_{b}(\lambda) A_{b}(\lambda) B_{c}(\lambda)+A_{a}(\lambda) A_{b}(\lambda) \\
=A_{a}(\lambda) A_{b}(\lambda)\left[1+A_{b}(\lambda) B_{c}(\lambda)\right] .
\end{gathered}
$$

On $\Lambda_{b}^{\prime}$, we have:

$$
\begin{gathered}
A_{a}(\lambda) B_{c}(\lambda)-A_{a}(\lambda) B_{b}(\lambda)= \\
A_{a}(\lambda) A_{b}(\lambda) A_{b}(\lambda) B_{c}(\lambda)+A_{a}(\lambda) A_{b}(\lambda)-2 A_{a}(\lambda) A_{b}(\lambda) \\
=A_{a}(\lambda) A_{b}(\lambda)\left[1+A_{b}(\lambda) B_{c}(\lambda)\right]-2 A_{a}(\lambda) A_{b}(\lambda) .
\end{gathered}
$$

Thus,

$$
P(a, c)-P(a, b)=\int_{\Lambda} A_{a}(\lambda) A_{b}(\lambda)\left[1+A_{b}(\lambda) B_{c}(\lambda)\right] d p(\lambda)-2 \int_{\Lambda_{b}^{\prime}} A_{a}(\lambda) B_{b}(\lambda) d p(\lambda) .
$$

Hence, we proved the following theorem:

Theorem 2 (Ryff [20]). (Generalization of the OB inequality for imperfect anti-correlations). Under assumption (12), the following inequality for classical correlations holds:

$$
|P(a, b)-P(a, c)|-P(b, c) \leq 1+2 \epsilon .
$$

By introducing the parameter $\gamma=1-\epsilon$, we write (15) as

$$
|P(a, b)-P(a, c)|-P(b, c) \leq 3-2 \gamma .
$$

By Theorem 1, we have the inequality: $3-2 \gamma<1,5$, i.e., $\gamma>3 / 4=0,75$. Thus, to be able to properly test the $\mathrm{OB}$ inequality, one has to be able to produce an ensemble of pairs of quantum systems in which the percentage of precisely (anti-) correlated pairs will be higher than

$$
\gamma=75 \% .
$$

\section{Original Bell Inequality: Taking into Account the Detection Efficiency}

For the $\mathrm{OB}$ inequality, the issue of the detection efficiency was studied in detail by J.-A. Larsson [21]. Here, we present similar consideration, but in slightly different form, which is consistent with the above presentation of the role of imperfection of correlations. Denote the set of hidden variables for which the pair $A_{a}(\lambda), B_{b}(\lambda)$ is detected by the symbol $\Gamma_{a b}$. The main parameter of the experimental interest is the probability of joint detection of a pair, $p\left(\Gamma_{a b}\right)$. For simplicity of considerations, we assume that this probability does not depend on the pair of settings, i.e.,

$$
\eta \equiv p\left(\Gamma_{a b}\right)
$$

Then, correlation conditioned on the pairwise detection is given by

$$
\tilde{P}(a, b)=\frac{1}{\eta} \int_{\Gamma_{a b}} A_{a}(\lambda) B_{b}(\lambda) d p(\lambda) .
$$


Theorem 3. Under the assumptions of $100 \%$ perfect anti-correlations and set-independent joint detection efficiency (see (17)), the following $O B$ inequality for detectable correlations holds:

$$
|\tilde{P}(a, b)-\tilde{P}(b, c)|-\tilde{P}(a, c) \leq \frac{(4-3 \eta)}{\eta} .
$$

Proof. From (18), we get: $\eta \tilde{P}(a, b)=P(a, b)-u_{a b}$, where

$$
u_{a b}=\int_{\Lambda \backslash \Gamma_{a b}} A_{a}(\lambda) B_{b}(\lambda) d p(\lambda)
$$

and, hence, $\left|u_{a b}\right| \leq(1-\eta)$. We have

$$
\begin{gathered}
\eta(|\tilde{P}(a, b)-\tilde{P}(b, c)|-\tilde{P}(a, c)) \\
\leq|P(a, b)-P(b, c)|-P(b, c)+3(1-\eta) \leq 1+3(1-\eta) .
\end{gathered}
$$

By dividing both sides of this inequality by $\eta$, we obtain (19).

To be able to violate inequality (19), the experimenter has to have sufficiently high the detection efficiency, such that $\frac{(4-3 \eta)}{\eta}<\frac{3}{2}$, i.e., $\eta>8 / 9=0.889$. Thus, the efficiency of the joint detection should be higher than $88.9 \%$. This result coincides with the corresponding result from ([21], p. 57). Thus, the detection efficiency should be higher than in the experimental tests for the CHSH inequality $[21,26]$.

\section{Original Bell Inequality: Taking into Account Imperfection of Anti-Correlations and the Detection Efficiency}

Theorem 4. Under the assumptions (17) and (12), the following experimentally testable version of the $O B$ inequality holds:

$$
|\tilde{P}(a, b)-\tilde{P}(b, c)|-\tilde{P}(a, c) \leq \frac{(4+2 \epsilon-3 \eta)}{\eta} .
$$

Proof. We have

$$
\begin{gathered}
\eta(|\tilde{P}(a, b)-\tilde{P}(b, c)|-\tilde{P}(a, c)) \leq|P(a, b)-P(b, c)|-P(b, c)+3(1-\eta) \\
\leq 1+2 \epsilon+3(1-\eta) .
\end{gathered}
$$

To be able to violate inequality (20), experimenter has to have sufficiently high anti-correlations and the detection efficiency, such that $\frac{4+2 \epsilon-3 \eta}{\eta}<\frac{3}{2}$. It is convenient to introduce a new parameter $\kappa=1-\epsilon$. Then, the generalized OB inequality has the form:

$$
|\tilde{P}(a, b)-\tilde{P}(b, c)|-\tilde{P}(a, c) \leq \frac{(6-2 \gamma-3 \eta)}{\eta}
$$

and the condition for possible violation can written as

$$
4 \gamma+9 \eta>12
$$

For example, let $\gamma=0.98$. Then, $\eta>0.9$. Thus, by approaching the $98 \%$ level of anti-correlations and the $90 \%$ level of detection efficiency, the experimenter can test the $\mathrm{OB}$ inequality. 


\section{Conclusions}

The modern quantum technology provides the sources producing photons in the singlet state with very high probability, up to $98 \%$ of generated ensemble of pairs. From this viewpoint, it is promising to perform the experimental test for the OB inequality by using entangled photons, $\mathrm{cf}$. with experiments $[11,12]$ to violate the $\mathrm{CHSH}$-like inequalities (the Eberhard and $\mathrm{CH}$ inequalities). However, as we have seen, tests for the $\mathrm{OB}$ inequality demand higher detection efficiency than tests for the CHSH inequality. We remind readers (see also [8] for discussion) that the detection efficiency is not reduced to the efficiency of photo-detectors. Although nowadays there are available photo-detectors having close to $100 \%$ efficiency, this does not solve the problem of the detection efficiency. A weak element of the experimental setup based on quantum optics is a polarization beam splitter, where one can lose $8-13 \%$ of photons. This loss can play a crucial role in attempts to lift the detection efficiency from $83 \%[21,26]$ in the tests for the $\mathrm{CHSH}$ inequality to approximately $90 \%$ in the planned experimental test for the $\mathrm{OB}$ inequality.

It may be reasonable to proceed under the assumption of fair sampling. In addition, such a project seems to be realizable.

If one wants to proceed without the fair sampling assumption, then it is very promising to test violation of the $\mathrm{OB}$ inequality by using entangled electron spins, i.e., the scheme of the Hensen et. al. [10] experiment that was done for the CHSH inequality. As was reported in [10], the parameter $\gamma$ in inequality (21) can be selected as $\gamma \approx 0.92$. It exceeds the bound $\gamma=0,75$ (see Equation (16)). Therefore, it seems that such an experiment can already be performed today.

Acknowledgments: The authors would like to thank A. Cabelo, E. Dzhafarov, G. Jager, J.-A. Larsson, S. Polyakov, K. Svozil, G. Weihs, S. Glancy, and F. De Martini for critical discussions and knowledge transfer; in particular, we are thankful to G. Jaeger for pointing us to Pitowsky's paper [9]. The first coauthor (AKH) was supported by the EU-project Quantum Information Access and Retrieval Theory (QUARTZ), Grant No. 721321.

Author Contributions: The paper is based on a long series of discussions between Andrei Khrennikov and Irina Basieva about the original Bell inequality versus the $\mathrm{CHSH}$ inequality. The text formalizing these discussions was written by A. Khrennikov.

Conflicts of Interest: The authors declare no conflict of interest.

\section{Appendix A}

Here, we follow the paper of Pitowsky [9]:

We recall that the Grothendieck constant of the order $n$ (denoted by $K_{G}(n)$ ) is defined as the least real number such that

$$
\left|\sum_{i=1}^{m} \sum_{j=1}^{m} a_{i j}\left\langle x_{i} \mid y_{j}\right\rangle\right| \leq K_{G}(n) \sup _{X_{i}, Y_{j}= \pm 1}\left|\sum_{i=1}^{m} \sum_{j=1}^{m} a_{i j} X_{i} Y_{j}\right|
$$

for every natural number $m$, every choice of real matrix elements $a_{i j}, i, j=1, \ldots, m$, and every choice of unit vectors $x_{1}, \ldots, x_{m}, y_{1}, \ldots y_{m} \in \mathbf{R}^{n}$. It was proved by Grothendieck that there exists

$$
K_{G}=\lim _{n \rightarrow \infty} K_{G}(n) .
$$

Tsirelson proved that $K_{G}(2)=\sqrt{2}$ if all vectors belong to the same plane; he also connected this result with the CHSH-inequality.

Tsirelson also proved the following theorem connecting the inequality (A1) to quantum theory:

Theorem A1. The following conditions on $m \times m$ matrix $\left(r_{i j}\right)$ are equivalent:

- There exists a finite-dimensional Hilbert space $H$ and Hermitian operators $A_{1}, \ldots, A_{m}$ and $B_{1}, \ldots, B_{m}$ acting in $H$ and having the spectrum in $[-1,+1]$, and a state $W$ on $H \otimes H$ such that $r_{i j}=\operatorname{Tr} W\left(\mathrm{~A}_{\mathrm{i}} \otimes \mathrm{B}_{\mathrm{j}}\right)$.

- $\quad$ There exist unit vectors $x_{1}, \ldots, x_{m}, y_{1}, \ldots y_{m} \in \mathbf{R}^{2 m}$ such that $r_{i j}=\left\langle x_{i} \mid y_{j}\right\rangle$. 
Thus, the fraction

$$
F_{\text {Tsirelson }} \equiv \sup _{m, a_{i j}, x_{i}, y_{j} \text { (unit vectors) }} \frac{\left|\sum_{i=1}^{m} \sum_{j=1}^{m} a_{i j}\left\langle x_{i} \mid y_{j}\right\rangle\right|}{\sup _{X_{i}, Y_{j}= \pm 1}\left|\sum_{i=1}^{m} \sum_{j=1}^{m} a_{i j} X_{i} Y_{j}\right|}
$$

can be used as a measure of quantumness (see also [27] and references herein for related studies about the "quantum/classical fraction".).

However, as was emphasized by Pitowsky [9], for the OB inequality, we can get the higher value for the "quantum/classical fraction" (see Theorem 1). (We remark that Theorem 1 is easily generalized to the Bell state: $\Psi=(|+-\rangle+|-+\rangle) / \sqrt{2}$.) This simple result stimulated Pitowsky to consider the general scheme for estimation of the "quantum/classical fraction" for perfectly correlated observables.

Define $A(n)$ as the least real number such that

$$
\sum_{1 \leq i<j \leq n} a_{i j}\left\langle x_{i} \mid x_{j}\right\rangle \leq A(n) \sup _{X_{i}= \pm 1} \sum_{1 \leq i<j \leq n} a_{i j} X_{i} X_{j}
$$

In contrast to the sequence of the Grothendieck constants $K_{G}(n)$, the sequence $A(n)$ is unbounded and $A(n)=O(\log n)$. Moreover, this is the best bound, i.e., there exists a positive constant $c$ such that

$$
\sum_{1 \leq i<j \leq n} a_{i j}\left\langle x_{i} \mid x_{j}\right\rangle>c \log n \sup _{X_{i}= \pm 1} \sum_{1 \leq i<j \leq n} a_{i j} X_{i} X_{j}
$$

for all $n$ and $a_{i j}$. Thus, the "quantum/classical fraction" approaches infinity! Finally, to connect this result with quantum physics, Pitowsky pointed to the following result following from Theorem A1:

Corollary A1. Given unit vectors $x_{1}, \ldots, x_{n} \in \mathbf{R}^{n}$, there exists a finite-dimensional Hilbert space $H$ and traceless Hermitian operators $A_{1}, \ldots, A_{n}$ and $B_{1}, \ldots, B_{n}$ acting in $H$ and having the spectrum \pm 1 , and a state $W$ on $H \otimes H$ such that $\operatorname{TrW}\left(\mathrm{A}_{\mathrm{i}} \otimes \mathrm{I}\right)=0, \operatorname{TrW}\left(\mathrm{I} \otimes \mathrm{B}_{\mathrm{j}}\right)=0$ and $\operatorname{TrW}\left(\mathrm{A}_{\mathrm{i}} \otimes \mathrm{B}_{\mathrm{j}}\right)=\left\langle\mathrm{x}_{\mathrm{i}} \mid \mathrm{y}_{\mathrm{j}}\right\rangle$.

We remark that these observables are perfectly correlated in the state $W$.

By Corollary A1, the fraction

$$
F_{\text {Pitowsky }}(n) \equiv=\sup _{a_{i j}, x_{i}(\text { unit vectors })} \frac{\sum_{1 \leq i<j \leq n} a_{i j}\left\langle x_{i} \mid x_{j}\right\rangle}{\sup _{X_{i}= \pm 1} \sum_{1 \leq i<j \leq n} a_{i j} X_{i} Y_{j}}
$$

can be used used as a measure of "quantumness".

Thus, by using the multidimensional analogs of the OB inequality, one can approach very high values of the "quantum/classical fraction". In addition, it is impossible to do this with CHSH-like inequalities.

\section{Appendix B. From the EPR Argument to the Original Bell Inequality}

The original Bell project can be formulated as the following:

- $\quad$ Einstein, Podolsky, and Rosen proved the existence of elements of reality (for the very special state).

- This implies that QM is not complete and it has to be considered as emergent from some theory with hidden variables.

- Einstein, Podolsky, and Rosen expected that such a theory would be local. (They did not construct such a theory, but they dreamed for it.)

- Bell's message based on violation of the OB inequality by (theoretical) quantum correlations: unfortunately, EPR realism is not compatible with locality.

We cite Bell ([1], p. 195): "Since we can predict in advance the result of measuring any chosen component of $\sigma_{2}$, by previously measuring the same component of $\sigma_{1}$, it follows that the result of 
any such measurement must actually be predetermined. Since the initial quantum mechanical wave function does not determine the result of an individual measurement, this predetermination implies the possibility of a more complete specification of the state." Thus, Bell's study was aimed at checking reazability the EPR project: to construct a subquantum model that would match statistical predictions of QM and at the same time describe the EPR elements of reality.

We remark that one could respond straightforwardly to EPR's argument by saying that measurement of the system without disturbance is impossible because a faster-than-light signal can move from $S_{1}$ to $S_{2}$. We remark that, for Einstein, Podolsky, and Rosen as well as Bohr, such explanation was not acceptable. In addition, this is the important point (We can mention Bohr's response to the EPR paper [28]. However, it seems that Bohr did not understand the EPR argument. In any event his reply does not explain the origin of perfect correlations.).

However, Bell proved (see [1], p. 199): "In a theory in which parameters are added to quantum mechanics to determine the results of individual measurements, without changing the statistical predictions, there must be a mechanism whereby the setting of one measuring device can influence the reading of another instrument, however remote. Moreover, the signal involved must propagate instantaneously, so that such a theory could not be Lorentz invariant." Thus, he treated violation of the $\mathrm{OB}$ inequality as the proof of nonlocality of any theory with hidden variables.

Now, we point to the crucial connection between the EPR argument and the OB inequality. For the singlet state (as for the original EPR state), spin projections are EPR's elements of reality. These elements per definition are equal to measurement outcomes (elements of reality for $S_{2}$ are measurement outcomes for $S_{1}$ ). Hence, values of variables of a subquantum theory beyond the singlet state can be identified with possible outcomes of measurements. Therefore, for the singlet state, subquantum and quantum correlations can be identified.

There are no reasons to assume this for a non-singlet state. Therefore, $\mathrm{CHSH}$-like projects that are not straightforwardly based on the perfect (anti-)correlations can be objected from the viewpoint that there is no reason to identify the values of subquantum and quantum variables and hence subquantum and quantum correlations. Subquantum correlations satisfy $\mathrm{CHSH}$-inequality, but quantum correlations violate it. (In particular, this was the viewpoint of De Broglie [29], see also [30] for details and references.). By rephrasing Bell, we can say "that what is proved, by impossibility proofs, is lack of imagination" of possible couplings beween subquantum and quantum correlations (cf. [31]).

Therefore, it is important to perform experimental tests for the OB inequality. This and only this test would imply that the issue of nonlocality has to be considered seriously.

\section{Appendix C. Interpretations of Violations of Bell Type Inequalities and Interpretations of Quantum Mechanics}

De Broglie's viewpoint [29] on interrelation between subquantum and quantum correlations (see Appendix B) can be generally formulated in the framework of the ontic-epistemic representation of quantum phenomena (see Atmanspacher and Primas [32]). This is the framework of the two-level description of natural phenomena. Besides an epistemic model representing outputs of measurements, one can consider an ontic model of reality as it is when nobody performs measurements. The quantum model is treated as an epistemic model (one of possible models describing knowledge that can be gained through measurements). Possible models with hidden variables are possible ontic models behind the quantum epistemic model.

We remark that the very common (especially among philosophers) statement that "an ontic model is about reality as it is" has to interpreted with caution. Scientists can speak only about models of reality, typically mathematical models. It may be better to follow Hertz, Boltzmann, and Schrödinger (see [33] and also [30]) and to speak about a theoretical model presenting a consistent picture of natural phenomena and an epistemic model representing the results of measurements. We remark that the quantum model cannot be considered as a theoretical model (in the sense of Hertz, Boltzmann, 
and Schrödinger), because, in particular, the measurement problem has not yet been solved (cf. with the claim of Allahverdyan, Balian, Nieuwenhuizen [34] that they solved this problem).

In such two-level framework, De Broglie's position [29] is justified. In general, there is no reason to identify the subquantum correlations with quantum ones, especially for the $\mathrm{CHSH}$-like inequalities. In particular, a theoretical model can be based on the continuous description of the field-type, cf. with "Einstein's dream" [35]. Subquantum correlations are correlations of such subquantum fields. Such correlations trivially violate Bell's inequality because the range of values of fields is unbounded. The concrete model of this type, prequantum classical statistical field theory (PCSFT), was developed in the series of works [31,36-38]. It generates correlations coinciding with the quantum correlations. (Here, a wave function determines the covariance operator of a prequantum random field.) The corresponding epistemic model is the threshold detection model [39].

We stress that generally interpretations of violation of Bell type inequalities are rigidly coupled to interpretations of the quantum mechanics (see, e.g., De Muynck [40,41], Fuchs [42,43], Fuchs and Schack [44], Grangier [45,46], 't Hooft [47,48], De Raedt et al. [49,50] Long, Qin, Yang et al. [51]), or more generally to interrelations between classical (Kolmogorovean) and quantum probabilities (see, e.g., Accardi [52,53], Ballentine [54-56], Khrennikov [22,25,57], Hess and Philipp [58], Hess [59]). Finally, we point to works of Khrennikov [60] and Kupczynski [61].

\section{References}

1. Bell, J. On the Einstein-Podolsky-Rosen paradox. Physics 1964, 1, 195-200. [CrossRef]

2. Bell, J. Speakable and Unspeakable in Quantum Mechanics; Cambridge University Press: Cambridge, UK, 1987.

3. Einstein, A.; Podolsky, B.; Rosen, N. Can quantum-mechanical description of physical reality be considered complete? Phys. Rev. 1935, 47, 777-780. [CrossRef]

4. Clauser, J.F.; Horne, M.A.; Shimony, A.; Holt, R.A. Proposed Experiment to Test Local Hidden-Variable Theories. Phys. Rev. Lett. 1969, 23, 880-884. [CrossRef]

5. Clauser, J.F.; Horne, M.A. Experimental consequences of objective local theories. Phys. Rev. D 1974, 10, 526-535. [CrossRef]

6. Clauser, J.F.; Shimony, A. Bell's theorem. Experimental tests and implications. Rep. Prog. Phys. 1978, 41, 1881-1927. [CrossRef]

7. Eberhard, P.H. Background level and counter efficiencies required for a loophole-free Einstein-Podolsky-Rosen experiment. Phys. Rev. A 1993, 47, R747-R750. [CrossRef]

8. Khrennikov, A.; Ramelow, S.; Ursin, R.; Wittmann, B.; Kofler, J.; Basieva, I. On the equivalence of the Clauser-Horne and Eberhard inequality based tests. Phys. Scr. 2014, T163, 014019. [CrossRef]

9. Pitowsky, I. New Bell inequalities for the singlet state: Going beyond the Grothendieck bound. J. Math. Phys. 2008, 49, 012101. [CrossRef]

10. Hensen, B.; Bernien, H.; Dréau, A.E.; Reiserer, A.; Kalb, N.; Blok, M.S.; Amaya, W. Experimental loophole-free violation of a Bell inequality using entangled electron spins separated by $1.3 \mathrm{~km}$. Nature 2015, 526, $682-686$. [CrossRef]

11. Giustina, M.; Versteegh, M.A.; Wengerowsky, S.; Handsteiner, J.; Hochrainer, A.; Phelan, K.; Amaya, W. A significant-loophole-free test of Bell's theorem with entangled photons. Phys. Rev. Lett. 2015, 115, 250401. [CrossRef]

12. Shalm, L.K.; Meyer-Scott, E.; Christensen, B.G.; Bierhorst, P.; Wayne, M.A.; Stevens, M.J.; Coakley, K.J. A strong loophole-free test of local realism. Phys. Rev. Lett. 2015, 115, 250402. [CrossRef]

13. Aspect, A.; Dalibard, J.; Roger, G. Experimental Test of Bell's Inequalities Using Time-Varying Analyzers. Phys. Rev. Lett. 1982, 49, 1804. [CrossRef]

14. Aspect, A. Three Experimental Tests of Bell Inequalities by the Measurement of Polarization Correlations between Photons; Orsay: Paris, France, 1983.

15. Weihs, G.; Jennewein, T.; Simon, C.; Weinfurter, R.; Zeilinger, A. Violation of Bell's Inequality under Strict Einstein Locality Conditions. Phys. Rev. Lett. 1998, 81, 5039-5043. [CrossRef]

16. Rowe, M.A.; Kielpinski, D.; Meyer, V.; Sackett, C.A.; Itano, W.M. Monroe, C.; Wineland, D.J. Experimental violation of a Bell's inequality with efficient detection. Nature 2001, 409, 791-794. [CrossRef] 
17. Giustina, M.; Mech, A.; Ramelow, S.; Wittmann, B.; Kofler, J.; Beyer, J.; Lita, A.; Calkins, B.; Gerrits, T.; Woo, N.S.; et al. Bell violation using entangled photons without the fair-sampling assumption. Nature 2013, 497, 227-230.

18. Christensen, B.G.; McCusker, K.T.; Altepeter, J.; Calkins, B.; Gerrits, T.; Lita, A.; Miller, A.; Shalm, L.K.; Zhang, Y.; Nam, S.W.; et al. Detection-Loophole-Free Test of Quantum Nonlocality, and Applications. Phys. Rev. Lett. 2013, 111, 1304-1306. [CrossRef]

19. Kupczynski, M. Can Einstein with Bohr debate on quantum mechanics be closed? arXiv 2016, arXiv:1603.00266. [CrossRef]

20. Ryff, L.C. Bell and Greenberger, Horne, and Zeilinger theorems revisited. Am. J. Phys. 1997, 65, 1197-1199.

21. Larsson, J.-A. Bells inequality and detector inefficiency. Phys. Rev. A 1998, 57, 3304-3305. [CrossRef]

22. Khrennikov, A. Interpretations of Probability, 2nd ed.; VSP Int. Sc. Publishers: Utrecht, The Netherlands; Tokyo, Japan, 1999; De Gruyter: Berlin, Germany, 2009, second edition, (completed). [CrossRef]

23. Khrennikov, A. Statistical measure of ensemble nonreproducibility and correction to Bell's inequality. Il Nuovo Cimento B 1999, 115, 179-184.

24. Khrennikov, A. A perturbation of $\mathrm{CHSH}$ inequality induced by fluctuations of ensemble distributions. J. Math. Phys. 2000, 41, 5934-5944.

25. Khrennikov, A. Frequency analysis of the EPR-Bell argumentation. Found. Phys. 2002, 32, 1159-1174. [CrossRef]

26. Garg, A.; Mermin, N.D. Detector inefficiencies in the Einstein-Podolsky-Rosen experiment. Phys. Rev. D 1987, 25, 3831-3835. [CrossRef]

27. Palazuelos, C. Super-activation of quantum non-locality. Phys. Rev. Lett. 2012, 109, 190401. [CrossRef]

28. Bohr, N. Can quantum-mechanical description of physical reality be considered complete? Phys. Rev. 1935, 48, 696-702. [CrossRef]

29. De Broglie, L. The Current Interpretation of Wave Mechanics: A Critical Study; Elsevier: Amsterdam, The Netherlands, 1964. [CrossRef]

30. Khrennikov, A. After Bell. Prog. Phys. 2017, 65, 1600014.

31. Khrennikov, A. Beyond Quantum; Pan Stanford Publ.: Singapore, 2014. [CrossRef]

32. Atmanspacher, H.; Primas, H. Epistemic and Ontic Quantumrealities. In Foundations of Probability and Physics-3; Adenier, G., Khrennikov, A.Y., Eds.; Ser. Conference Proceedings 750; American Institute of Physics: Melville, NY, USA, 2005; pp. 49-62.

33. D'Agostino, S. Continuity and completeness in physical theory: Schrödinger's return to the wave interpretation of quantum mechanics in the 1950's. In E. Schrödinger: Philosophy and the Birth of Quantum Mechanics; Editions Frontieres; Bitbol, M., Darrigol, O., Eds.; Centre National de la Recherche Scientifique: Gif-sur-Yvette, Paris, France, 1992; pp. 339-360.

34. Allahverdyan, A.E. Balian, R.; Nieuwenhuizen, T.M. Understanding quantum measurement from the solution of dynamical models. Phys. Rep. 2013, 525, 1-166.

35. Einstein, A.; Infeld, L. Evolution of Physics: The Growth of Ideas from Early Concepts to Relativity and Quanta; Simon and Schuster: New York, NY, USA, 1961. [CrossRef]

36. Khrennikov, A. Nonlinear Schrdinger equations from prequantum classical statistical field theory. Phys. Lett. A 2006, 357, 171-176.

37. Khrennikov, A. Quantum mechanics as the quadratic Taylor approximation of classical mechanics: The finite-dimensional case. Theor. Math. Phys. 2007, 152, 1111-1121. [CrossRef]

38. Khrennikov, A. To quantum averages through asymptotic expansion of classical averages on infinite-dimensional space. J. Math. Phys. 2007, 48, 013512. [CrossRef]

39. Khrennikov, A. Quantum probabilities and violation of $\mathrm{CHSH}$-inequality from classical random signals and threshold type detection scheme. Prog. Theor. Phys. 2012, 128, 31-58. [CrossRef]

40. De Muynck, W.M. Interpretations of quantum mechanics, and interpretations of violations of Bell's inequality. In Foundations of Probability and Physics; Khrennikov, A.Y., Ed.; Series PQ-QP: Quantum Probability and White Noise Analysis 13; WSP: Singapore, 2001; pp. 95-104. [CrossRef]

41. De Muynck, W.M. Foundations of Quantum Mechanics, an Empiricists Approach; Kluwer Academic Publ.: Dordrecht, The Netherlands, 2002. 
42. Fuchs, C.A. Quantum mechanics as quantum information (and only a little more). In Quantum Theory: Reconsideration of Foundations; Khrennikov, A. Ed.; Ser. Math. Modeling 2; Växjö University Press: Växjö, Sweden, 2002; pp. 463-543.

43. Fuchs, C. Delirium quantum (or, where I will take quantum mechanics if it will let me). In Foundations of Probability and Physics-3; Adenier, G., Fuchs, C., Khrennikov, A.Y., Eds.; Ser. Conference Proceedings 889; American Institute of Physics: Melville, NY, USA, 2007; pp. 438-462.

44. Fuchs, C.A.; Schack, R. QBism and the Greeks: Why a quantum state does not represent an element of physical reality. Phys. Scr. 2014, 90, 015104.

45. Grangier, P. Contextual objectivity: A realistic interpretation of quantum mechanics. Eur. J. Phys. 2002, $23,331$. [CrossRef]

46. Grangier, P. Contextual objectivity and the quantum formalism. Int. J. Quantum Inf. 2005, 3, 17-22. [CrossRef]

47. Hooft, G. The free-will postulate in quantum mechanics. arXiv 2007, arXiv:quant-ph/0701097v1. [CrossRef]

48. Hooft, G. The cellular automaton interpretation of quantum mechanics. arXiv 2014, arXiv:1405.1548. [CrossRef]

49. De Raedt, H.; Katsnelson, M.I.; Michielsen, K. Quantum theory as the most robust description of reproducible experiments. Ann. Phys. 2014, 347, 45-73.

50. De Raedt, H.; Katsnelson, M.I.; Donker, H.C.; Michielsen, K. Quantum theory as a description of robust experiments: Derivation of the Pauli equation. Ann. Phys. 2015, 359, 166-186. [CrossRef]

51. Long, G.L.; Qin, W.; Yang, Z.; Li, J.L. Realistic interpretation of quantum mechanics and encounter-delayedchoice experiment. Sci. China Phys. Mech. Astron. 2018, 61, 030311. [CrossRef]

52. Accardi, L. The probabilistic roots of the quantum mechanical paradoxes. In The Wave-Particle Dualism. A Tribute to Louis de Broglie on his 90th Birthday; Diner, S., Fargue, D., Lochak, G., Selleri, F., Eds.; D. Reidel Publ. Company: Dordrecht, The Netherlands, 1984; pp. 47-55. [CrossRef]

53. Accardi, L. Some loopholes to save quantum nonlocality. In Foundations of Probability and Physics-3; Adenier, G., Khrennikov, A.Y., Eds.; Ser. Conference Proceedings 750; American Institute of Physics: Melville, NY, USA, 2005; pp. 1-20. [CrossRef]

54. Ballentine, L.E. The statistical interpretation of quantum mechanics. Rev. Mod. Phys. 1989, 42, 358-381.

55. Ballentine, L.E. Quantum Mechanics; Prentice Hall: Englewood Cliffs, NJ, USA, 1989.

56. Ballentine, L.E. Interpretations of probability and quantum theory. In Foundations of Probability and Physics, Quantum Probability and White Noise Analysis; Khrennikov, A.Y., Ed.; WSP: Singapore, 2001; Volume 13, pp. 71-84. [CrossRef]

57. Khrennikov, A. Contextual viewpoint to quantum stochastics. J. Math. Phys. 2003, 44, 2471-2478.

58. Hess, K.; Philipp, W. Bell's theorem: Critique of proofs with and without inequalities. In Foundations of Probability and Physics-3; Adenier, G., Khrennikov, A.Y., Eds.; Ser. Conference Proceedings 750; American Institute of Physics: Melville, NY, USA, 2005; pp. 150-155.

59. Hess, K. Einstein Was Right; Pan Stanford Publ.: Singapore, 2014.

60. Khrennikov, A. CHSH inequality: Quantum probabilities as classical conditional probabilities. Found. Phys. 2015, 45, 711-725. [CrossRef]

61. Kupczynski, M. Bell inequalities, experimental protocols and contextuality. Found. Phys. 2015, 45, 735-753.

(C) 2018 by the authors. Licensee MDPI, Basel, Switzerland. This article is an open access article distributed under the terms and conditions of the Creative Commons Attribution (CC BY) license (http://creativecommons.org/licenses/by/4.0/). 